\title{
Could PET imaging provide insights into Zika virus neurological sequelae progression?
}

\author{
Darci R Smith*,1 \& Thomas M Bocan² \\ ${ }^{1}$ Virology Division, US Army Medical Research Institute of Infectious Diseases, 1425 Porter St, Ft Detrick, MD 21702, USA \\ ${ }^{2}$ Molecular \& Translational Sciences Division, US Army Medical Research Institute of Infectious Diseases, 1425 Porter St, Ft Detrick, \\ MD 21702, USA \\ * Author for correspondence: darci.r.smith.ctr@mail.mil
}

\begin{abstract}
“An in vivo imaging approach is particularly advantageous to study progression of ZIKV-induced neurological sequelae because the development of neuropathology and congenital defects is a dynamic process."
\end{abstract}

First draft submitted: 9 November 2017; Accepted for publication: 21 November 2017; Published online: 10 January 2018

Keywords: FDG • FMISO • molecular imaging • PET • viral bioreporter • Zika virus

Zika virus (ZIKV) is a mosquito-borne flavivirus that has emerged since 2007 causing human outbreaks in Africa, Asia, Oceania and most recently in the Americas. Mortality is not a common feature of human ZIKV infection, which is generally asymptomatic and self-limiting in most individuals. However, during the recent epidemics in Asia and the Americas, more severe clinical complications have been reported. Severe disease is characterized by neurological complications, which in adults include Guillain-Barré syndrome or in a few cases, encephalopathy, meningoencephalitis and acute myelitis [1]. ZIKV infection in pregnant women is a major global public health concern due to its link to congenital abnormalities including microcephaly, spontaneous abortion and intrauterine growth restriction [2], now referred to as congenital Zika syndrome.

The unexpectedly frequent and severe clinical complications of ZIKV infection have prompted intense research on host-virus interactions, which remains a high priority research area. It is crucial that we characterize the neuropathogenesis and causative roles of ZIKV infection in the etiology of fetal microcephaly and other congenital malformations during pregnancy. Appropriate use of animal models is needed to address some of these key unanswered questions regarding the pathogenesis of ZIKV and to evaluate candidate medical countermeasures. Current approaches to study viral pathogenesis involve reduced mortality or extended time-to-death as the efficacy end point, viremia as an index of infection and serial necropsies to establish the pathological consequences of infection. New technology in the form of molecular imaging tools shifts the classical paradigm of serial necropsies and offers less invasive alternatives capable of providing a dynamic assessment of pathogen infection in real time. An in vivo imaging approach is particularly advantageous to study progression of ZIKV-induced neurological sequelae because the development of neuropathology and congenital defects is a dynamic process. While molecular imaging has been used to characterize disease progression and evaluate drugs in the areas of neuroscience, cardiovascular, inflammation and oncology, application of imaging in infectious disease is limited [3].

We recently described the ability and sensitivity of PET imaging using $\left[{ }^{18} \mathrm{~F}\right] \mathrm{DPA}-714$, a translocator protein (TSPO) $18 \mathrm{kDa}$ radioligand, to detect and quantify neuroinflammation in ZIKV-infected mice [4]. Our results demonstrated that global neuroinflammation plays an important role in the progression of ZIKV infection in a transiently immunosuppressed mouse model [5]. Importantly, the use of [ $\left.{ }^{18} \mathrm{~F}\right] \mathrm{DPA}-714$ PET imaging was more sensitive compared with standard histology techniques for the detection of neuroinflammation. The significant increase in global neuroinflammation occurred despite limited regional detection of viral RNA suggesting that the host inflammatory response is associated with the ZIKV-induced neuropathogenesis, which is not solely caused by virus-mediated damage. A major advantage of this molecular imaging approach is the ability to dynamically assess pathology in the intact animal. Additionally, other molecular imaging probes are available to characterize the host response to virus infection were not addressed in this initial study. For example, tracers are available to evaluate 
metabolisms, hypoxia and general vascular dysfunction to include blood-brain barrier integrity and perfusion. Other imaging modalities such as single-photon emission computed tomography and MRI could be used to provide additional insight into the neuropathology, for example, demyelination and neuronal function/connectivity. Below we discuss possible ways molecular imaging by PET may be a useful tool to assess the role of key biological processes in the pathophysiology of ZIKV-induced neurological sequelae.

\section{Role of the placenta in the development of fetal malformations}

Animal and in vitro models have helped provide a better understanding of the pathogenic mechanisms of ZIKV. Mouse models have addressed the causal relationship between ZIKV infection during pregnancy and pathology in the fetuses [6-9]. In several studies, pregnant mice were infected through peripheral inoculation of ZIKV and demonstrated vertical transmission resulting in fetal demise with significant brain abnormalities $[6,8,9]$. These studies suggest that ZIKV gains access to the fetus after crossing the placental barrier, which is composed of different types of trophoblasts and ancillary cells. ZIKV most likely replicates in subgroups of trophoblasts, fetal endothelial cells and Hofbauer placental macrophages based on the mouse models and recent studies of infection in primary human cells and tissue samples [10,11]. Additionally, the neurotropism of ZIKV has been demonstrated by in vitro and in vivo experiments where neural stem cells, brain organoids and embryonic mouse brains were infected resulting in apoptosis and dysregulation of growth [7,12-14]. Collectively, these studies established ZIKV infection in fetuses with resultant injury to cells in the CNS. However, it is unknown to what degree placental insufficiency contributes to fetal malformations or growth retardation. ZIKV infection could lead to inflammation and severe placental vascular damage including a reduction in fetal blood vessels, blood flow or glucose metabolism. Molecular imaging using 2-deoxy-2- $\left[{ }^{18} \mathrm{~F}\right]$ fluoro-D-glucose $\left(\left[{ }^{18} \mathrm{~F}\right]-\mathrm{FDG}\right)$ PET has shown that the placenta has a twofold higher avidity for glucose than the maternal or fetal brain [15]. If ZIKV causes placental damage which alters the delivery of glucose to the fetal brain, $\left[{ }^{18} \mathrm{~F}\right]$-FDG PET could be one method for monitoring the observed growth effects. Changes in placental blood flow could be assessed using ${ }^{15} \mathrm{O}$-water or performing an acute dynamic PET scan with a focus on the placenta following an $\left[{ }^{18} \mathrm{~F}\right]-\mathrm{FDG}$ injection.

\section{Infection of the fetal brain \& development of neuropathology}

ZIKV could cross the placental barrier without excessive damage and infect the fetal brain preferentially through neural progenitor cells. Key aspects of neuronal damage are poorly understood and it is currently unclear how ZIKV infection leads to death of neurons. Neuronal injury and death can be caused by direct viral infection and/or initiated by virus-induced inflammatory response. Molecular imaging by PET would help provide important insight into this mechanism by tracking the uptake and replication of ZIKV in the fetal brain. Numerous PET amenable bioreporters which can be engineered into the virus have been reported in the literature. The most often used reporter is herpes simplex virus thymidine kinase (HSV-tk1, 1128 nucleotides) with [ $\left.{ }^{124} \mathrm{I} /{ }^{18} \mathrm{~F}\right]$-29-fluoro-29deoxy-1-b-D-arabinofuranosyl-5-iodouracil $\left(\left[{ }^{124} \mathrm{I} /{ }^{18} \mathrm{~F}\right]\right.$-FIAU) as the enzyme substrate and PET as the imaging modality [16]. The viral thymidine kinase is translated in the cell along with the viral RNA and phosphorylates the exogenously delivered radiolabeled $\left[{ }^{124} \mathrm{I} /{ }^{18} \mathrm{~F}\right]$-FIAU substrate, trapping it in cells, and thereby labeling cells which have been infected with the virus.

The assessment of direct virus replication can be coupled with studies to assess the indirect effects of virus infection by using specific PET tracers to monitor key biological processes such as inflammation and metabolism. For example, molecular imaging by PET could be used to assess the effects of ZIKV on fetal brain $\left[{ }^{18} \mathrm{~F}\right]$-FDG metabolism. $\left[{ }^{18} \mathrm{~F}\right]$ FDG has routinely been used to assess tissue metabolism. As assessed by PET, reductions in glucose metabolism have been noted in Alzheimer's disease and mild cognitive impairment [17] and the hypometabolism is believed to be associated with reduced neuronal activity and neuronal loss [18,19]. If the growth retardation and altered metabolism is associated with tissue hypoxia, $\left[{ }^{18} \mathrm{~F}\right]$ fluoromisonidazole $\left(\left[{ }^{18} \mathrm{~F}\right] \mathrm{FMISO}\right)[20]$ is a selective measure for assessing hypoxia. In viable cells that are hypoxic, the PET tracer is reduced to a reactive intermediate by cellular reductases which covalently bind to thiol groups of intracellular proteins and thereby accumulate in areas of hypoxia.

\section{Development \& evaluation of medical countermeasures}

Molecular imaging technology provides a novel approach to evaluate the efficacy of medical countermeasures. For example, molecular imaging by PET can be used to dynamically assess the efficacy of a drug or vaccine's effect on virus replication, tissue distribution and host response. Additionally, the biodistribution of radiolabeled drugs can be determined in real time. By tracking viral replication, one can map the progression of the disease and correlate 
changes in tissue pathology using additional molecular imaging probes with tissue viral load. Unlike current methods which monitor plasma viral load as an index of infection, measuring tissue viral levels by imaging more directly assesses the degree of infection. In addition, when evaluating tissue levels of a PET radiolabeled drug, a relationship between plasma and tissue drug and virus levels can be established to better direct therapeutic intervention. Linking measures of tissue inflammation, metabolism, hypoxia, necrosis, apoptosis and vascular damage with tissue viral load over time can also provide mechanistic information relating to viral stimulus and tissue response. At present it is important to utilize the available imaging probes to perform studies where samples from the same animals can be taken for proteomic and genomic analysis. These samples can then be used to identify biomarkers of infection and disease progression and be evaluated in relation to a specific stage of the disease.

In conclusion, many questions remain unanswered about the mechanisms by which ZIKV might cause severe neurological sequelae including congenital defects. A better understanding of how the virus is vertically transmitted and gains access to the developing nervous system is critical. With the development of new molecular imaging probes, some of which may be virus specific, and application of additional imaging approaches like MRI, it is possible to characterize the disease more thoroughly, providing both structural and functional information in a dynamic fashion within the same animal to aid in therapeutic drug discovery efforts.

\section{Disclosure}

Opinions, interpretations, conclusions and recommendations stated within the article are those of the authors and not necessarily endorsed by the US Army.

\section{Financial \& competing interests disclosure}

The authors have no relevant affiliations or financial involvement with any organization or entity with a financial interest in or financial conflict with the subject matter or materials discussed in the manuscript. This includes employment, consultancies, honoraria, stock ownership or options, expert testimony, grants or patents received or pending, or royalties.

No writing assistance was utilized in the production of this manuscript.

\section{References}

1 Li H, Saucedo-Cuevas L, Shresta S, Gleeson JG. The neurobiology of Zika virus. Neuron 92(5), 949-958 (2016).

2 Brasil P, Pereira JP Jr, Raja Gabaglia C et al. Zika virus infection in pregnant women in Rio de Janeiro: preliminary report. N. Engl. J. Med. 375(24), 2321-2334 (2016).

3 Bocan TM, Panchal RG, Bavari S. Applications of in vivo imaging in the evaluation of the pathophysiology of viral and bacterial infections and in development of countermeasures to BSL3/4 pathogens. Mol. Imaging Biol. 17(1), 4-17 (2015).

4 Kuszpit K, Hollidge BS, Zeng X et al. [18F]DPA-714 PET imaging reveals global neuroinflammation in Zika virus-infected mice. Mol. Imaging Biol. doi:10.1007/s11307-017-1118-1112 (2017) (Epub ahead of print).

5 Smith DR, Hollidge B, Daye S et al. Neuropathogenesis of Zika virus in a highly susceptible immunocompetent mouse model after antibody blockade of type I interferon. PLoS Negl. Trop. Dis. 11(1), e0005296 (2017).

6 Cugola FR, Fernandes IR, Russo FB et al. The Brazilian Zika virus strain causes birth defects in experimental models. Nature 534(7606), 267-271 (2016).

$7 \quad \mathrm{Li}$ C, Xu D, Ye Q et al. Zika virus disrupts neural progenitor development and leads to microcephaly in mice. Cell Stem Cell 19(5), 672 (2016).

8 Miner JJ, Cao B, Govero J et al. Zika virus infection during pregnancy in mice causes placental damage and fetal demise. Cell 165(5), 1081-1091 (2016).

9 Wu KY, Zuo GL, Li XF et al. Vertical transmission of Zika virus targeting the radial glial cells affects cortex development of offspring mice. Cell Res. 26(6), 645-654 (2016).

10 Bayer A, Lennemann NJ, Ouyang Y et al. Type III interferons produced by human placental trophoblasts confer protection against Zika virus infection. Cell Host Microbe 19(5), 705-712 (2016).

11 Quicke KM, Bowen JR, Johnson EL et al. Zika virus infects human placental macrophages. Cell Host Microbe 20(1), 83-90 (2016).

12 Dang J, Tiwari SK, Lichinchi G et al. Zika virus depletes neural progenitors in human cerebral organoids through activation of the innate immune receptor TLR3. Cell Stem Cell 19(2), 258-265 (2016).

13 Garcez PP, Loiola EC, Madeiro Da Costa R et al. Zika virus impairs growth in human neurospheres and brain organoids. Science 352(6287), 816-818 (2016).

14 Tang H, Hammack C, Ogden SC et al. Zika virus infects human cortical neural progenitors and attenuates their growth. Cell Stem Cell 18(5), 587-590 (2016). 
15 Sawatzke AB, Norris AW, Spyropoulos F et al. PET/CT imaging reveals unrivaled placental avidity for glucose compared with other tissues. Placenta 36(2), 115-120 (2015).

16 Brader P, Serganova I, Blasberg RG. Noninvasive molecular imaging using reporter genes. J. Nucl. Med. 54(2), 167-172 (2013).

17 Rini JN, Bhargava KK, Tronco GG et al. PET with FDG-labeled leukocytes versus scintigraphy with 111In-oxine-labeled leukocytes for detection of infection. Radiology 238(3), 978-987 (2006).

18 Lewis DY, Soloviev D, Brindle KM. Imaging tumor metabolism using positron emission tomography. Cancer J. 21(2), 129-136 (2015).

19 Mosconi L. Brain glucose metabolism in the early and specific diagnosis of Alzheimer's disease. FDG-PET studies in MCI and AD. Eur. J. Nuclear Med. Mol. Imaging 32(4), 486-510 (2005).

20 Clark PM, Flores G, Evdokimov NM et al. Positron emission tomography probe demonstrates a striking concentration of ribose salvage in the liver. Proc. Natl Acad. Sci. USA 111(28), e2866-e2874 (2014). 